\title{
Effect of Varying the Semiconducting/Metallic Tube Ratio on the Performance of Mixed Single-Walled Carbon Nanotube Network Gas Sensors
}

\author{
Sung Joon Min, ${ }^{1}$ Jin Woong Kim, ${ }^{2}$ Joon Hyub Kim, ${ }^{2}$ Joon Hyock Choi, ${ }^{3}$ \\ Chan Won Park, ${ }^{4}$ and Nam Ki Min ${ }^{2}$ \\ ${ }^{1}$ Department of Biomicrosystem Technology, Korea University, Seoul 02841, Republic of Korea \\ ${ }^{2}$ Department of Electro-Mechanical Systems Engineering, Korea University, Sejong 30019, Republic of Korea \\ ${ }^{3}$ Department of Electrical Engineering, Suwon Science College, Hwaseong 18516, Republic of Korea \\ ${ }^{4}$ Department of Electrical and Electronics Engineering, Kangwon National University, Chuncheon 24341, Republic of Korea
}

Correspondence should be addressed to Chan Won Park; cwpark@kangwon.ac.kr and Nam Ki Min; nkmin@korea.ac.kr

Received 31 January 2017; Revised 17 May 2017; Accepted 24 May 2017; Published 28 June 2017

Academic Editor: Yasuhiko Hayashi

Copyright (c) 2017 Sung Joon Min et al. This is an open access article distributed under the Creative Commons Attribution License, which permits unrestricted use, distribution, and reproduction in any medium, provided the original work is properly cited.

\begin{abstract}
The sensing properties of mixed networks consisting of semiconducting and metallic single-walled carbon nanotubes (SWCNTs) have been found to largely vary depending on the ratio of semiconducting to metallic tubes. Solution-deposited $99 \%$ semiconductor-enriched nanotube networks exhibited a sensitivity of $1.908 \% / \mathrm{ppm}$, whereas the unenriched $66 \%$ and $90 \%$ enriched samples exhibited a sensitivity of $0.027 \% / \mathrm{ppm}$ and $0.113 \% / \mathrm{ppm}$, respectively. These results suggest that it is extremely important to minimize the metallic pathways to achieve high sensitivity. After an oxygen plasma treatment, the unenriched $66 \%$ sample exhibited a $526 \%$ increase in sensitivity $(0.142 \% / \mathrm{ppm})$ compared to the untreated one, whereas the $90 \%$ device demonstrated a sensitivity of $1.521 \% / \mathrm{ppm}$, which corresponds to an improvement in the sensitivity of 13.5 times the pristine $90 \%$ sample. In addition, the plasmatreated sensors exhibited a much faster response time than the untreated one. The significant improvement in the performance of the highly enriched network sensors was explained by the large increase in the anchoring sites for ammonia molecules on the surface of the semiconducting single-walled CNTs and the faster charge transfer from absorbed molecules.
\end{abstract}

\section{Introduction}

Chemical and biological sensors have emerged as dynamic approaches for detecting specific analytes for environmental protection, medical diagnostics, food and industrial safety, and security. Such devices and systems are becoming an increasingly indispensable part of our daily lives because the detection or identification of unknown gases and vapors is critically important for improving and protecting human health, safety, quality of life, and the environment [1]. Nanotechnology is expected to lead to the development of inexpensive, simple sensors or devices that can rapidly detect, identify, and quantify biological and chemical species $[2,3]$. Owing to the diverse nature of their potential applications, such nanosensors could significantly impact multiple sectors of the economy including healthcare, pharmaceutical, agricultural, food, environmental, and consumer products [2-5].

One of the most successful nanosensors is based on carbon nanotube (CNT) networks. Randomly oriented or aligned networks of single-walled CNTs (SWCNTs) are a promising material for future nanoscale functional devices such as chemical and biological sensors [4, 68]. SWCNT-network-based sensors have been categorized into three groups: modification of the Schottky barrier at the metal/CNT interface $[9,10]$; charge transfer between a nanotube and an adsorbed analyte [11, 12]; and capacitive gating of the nanotube $[13,14]$. Although it does not matter which sensing mechanism is actually responsible for the operation of a SWCNT sensor, it is extremely important to have a percolative network of semiconducting (s) SWCNTs 
to achieve high performance because only s-SWCNTs exhibit significant changes in their electrical properties (conductance) due to the presence of absorbed molecules $[15,16]$. For mixtures of metallic $(\mathrm{m})$ and semiconducting nanotubes, $\mathrm{m}$ SWCNTs can deteriorate the sensor performance owing to two effects. Namely, metallic tubes are relatively insensitive to their chemical environment and their interactions with other species [15] and electrically short the s-SWCNTs and the device if they form a percolating pathway between two electrodes. Thus, the electrical performance of SWCNTnetwork-based sensors is strongly influenced by the semiconducting/metallic tube ratio $(S / M)$ within the SWCNT films.

Another factor that affects the sensitivity of the CNT sensors is the oxygenated functional groups attached to the sidewall of the SWCNT tubes. These oxygen-containing groups are found to significantly increase the interaction of CNTs with gas molecules $[4,17-20]$. The CNT surface can be modified by changing its chemical composition using chemical and plasma treatments [21-23]. Both treatments introduce oxygenated functional groups into the SWCNTs but produce different effects on the metallic and semiconducting SWCNT surfaces $[21,24]$.

The typical SWCNT-network system is a mixture containing approximately one-third m-SWCNTs and two-thirds s-SWCNTs. This inhomogeneity often results in fabrication complexity, low sensitivity, and poorly reproducible sensor performance. Recently, semiconductor-enriched nanotubes have become commercially available, which makes it easy for research groups to explore device applications based on enriched CNT networks [25]. Several experimental and theoretical studies have demonstrated excellent field-effect transistors (FETs) with a high on-state conductance and high on/off ratios using semiconductor-enriched CNT networks [26-28], but there have been few reports to date on the effects of $S / M$ and plasma treatments on the performance of semiconductor-enriched SWCNT-network sensors.

In this study, we fabricate ammonia gas sensors based on SWCNTs with different semiconducting content (typical SWCNT and 90\% enriched and 99\% enriched SWCNTs) and demonstrate the large improvement in the sensitivity to $\mathrm{NH}_{3}$ gas as a function of $S / M$. Finally, the influence of the $\mathrm{O}_{2}$ plasma treatment on the static and dynamic performance of the highly semiconductor-enriched SWCNTnetwork sensors is evaluated using ammonia gas as a target analyte.

\section{Materials and Methods}

2.1. Fabrication of Ammonia Gas Sensors. SWCNT-network sensors with $3 \mathrm{~mm} \times 8 \mathrm{~mm}$ size (active area $=9 \mathrm{~mm}^{2}$ ) were fabricated on glass substrates. The SWCNT networks used as chemiresistors were deposited on a 4-inch glass wafer, and interdigitated palladium electrodes were then deposited on top of the SWCNT-network. The normal unenriched SWCNT material was purchased from HanWha Nanotech (Korea) and consisted of 60-70 vol\% SWCNTs produced by the arc discharge method using a Ni-Y catalyst. In addition, $90 \%$ and $99 \%$ semiconductor-enriched SWCNTs were obtained from NanoIntegris (USA). The SWCNT networks were deposited by using a solution method [29, 30]. First, the glass wafer was oxygen-plasma-treated and functionalized with amine-containing molecules (poly-L-lysine). The semiconductor-enriched SWCNT solution was subsequently dispensed onto the self-assembled-monolayer-modified surface and dried for $2 \mathrm{~h}$. Atomic force microscopy (AFM) was used to record the topography and to measure the SWCNT film thickness. The typical SWCNT thickness derived from AFM line profiles was approximately $30 \mathrm{~nm}$. Submonolayer films instead of continuous networks were formed for the 90\% and 99\% SWCNTs, as shown in Figures 1(c) and 1(d). Figure S1 (in Supplementary Material available online at https://doi.org/10.1155/2017/8761064) is a larger version of the SEM images for SWCNT shown in Figure 1. Next, for the interdigitated electrode and contact pad, a $50 \mathrm{~nm}$ thick palladium film was evaporated onto the SWCNT networks by a thermal evaporation system and patterned by lift-off with optical lithography using a negative photoresist. Finally, the SWCNT films were patterned using oxygen plasma etching.

2.2. Oxygen Plasma Treatment for Surface Functionalization. After patterning, some SWCNT networks were radiofrequency (RF) plasma-treated with oxygen gas under much lower plasma power than those applied during etching. The typical parameters optimized through several experiments were an $\mathrm{O}_{2}$ flow rate of $10 \mathrm{sccm}$, a substrate temperature of $25^{\circ} \mathrm{C}$, and an RF power of $10 \mathrm{~W}$ for $10 \mathrm{~s}$.

The sensors are labeled as unenriched $66 \%, 90 \%$ enriched, and $99 \%$ enriched, corresponding to the normal SWCNTs and the $90 \%$ and $99 \%$ semiconductor-enriched SWCNT networks, respectively, and the plasma-treated sensor devices are labeled as p- $66 \%$ and p-90\% enriched, respectively. The plasma-treated $99 \%$ enriched device was not tested because the SWCNT-network was completely etched away, even under very low power $\mathrm{O}_{2}$ plasma.

2.3. Measurement. The sensors were placed in the test fixture attached to a gas manifold that had the capability of mixing several gases using nitrogen as the carrier gas. A detailed description of the flow system and resistance measurement is presented elsewhere [31]. An LCR meter (test signal: $20 \mathrm{kHz}$ and $1 \mathrm{~V}_{\mathrm{rms}}$ ) was used to measure the ammonia gas-sensing properties of different SWCNT networks as a function of the ammonia gas concentration levels in a chamber with electrical feed-through. The $\mathrm{NH}_{3}$ gas concentration was varied in the range from $3.6 \mathrm{ppm}$ to $64.2 \mathrm{ppm}$. Values with error bars (see Figures 3, 4, 5, and 7) represent the average and standard deviations of triplicate measurements performed on independently fabricated electrodes.

\section{Results and Discussion}

3.1. I-V Characteristics of SWCNT Networks with $\mathrm{NH}_{3}$ Gas. The assembled devices from all three SWCNT networks were first tested to confirm the formation of ohmic contacts and stability under ambient conditions and were then exposed to $\mathrm{NH}_{3}$ at different concentrations to demonstrate sensor performance. 
(b) $66 \%$ SWCNT

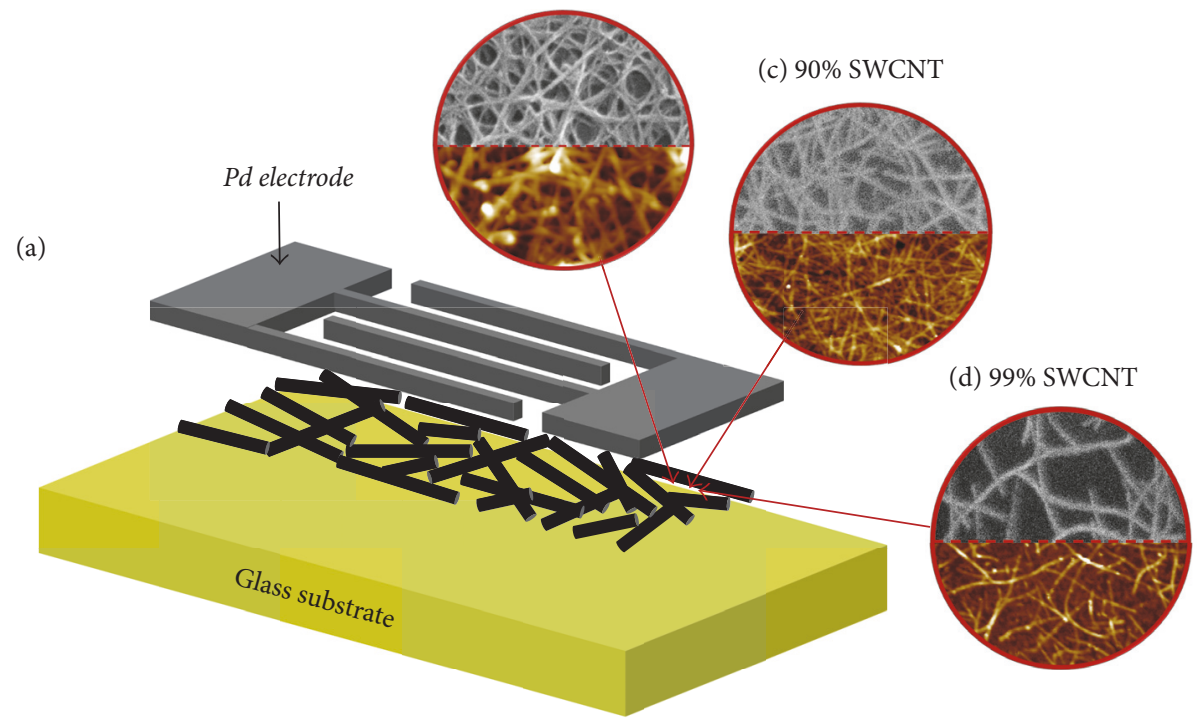

FIGURE 1: (a) Schematic illustration of the SWCNT-network-based $\mathrm{NH}_{3}$ gas sensor with an interdigitated electrode. SEM (upper) and AFM (lower) images of (b) unenriched 66\%, (c) 90\% enriched, and (d) 99\% enriched SWCNT networks.

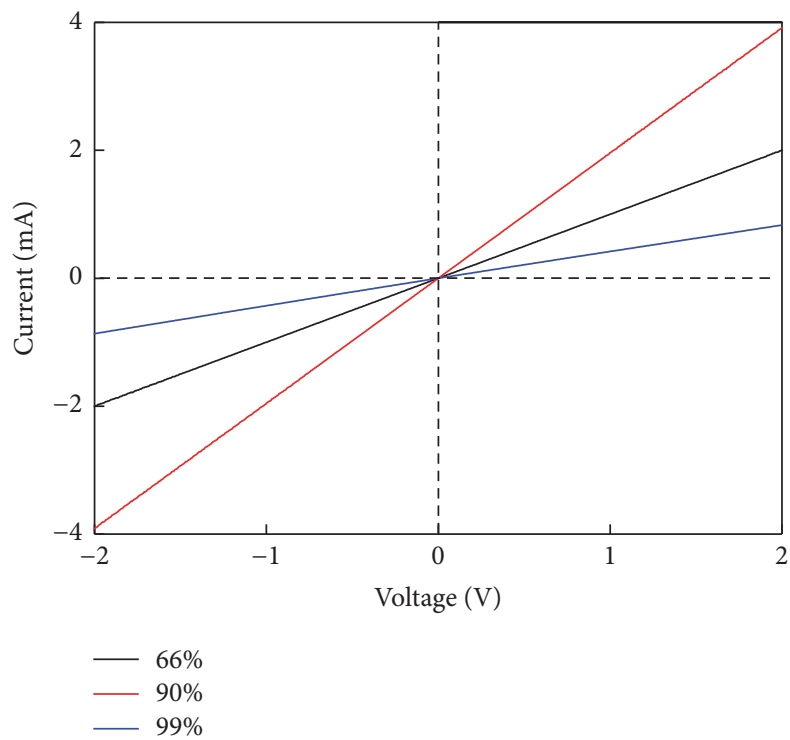

Figure 2: Current-voltage characteristics measured before exposing the unenriched $66 \%, 90 \%$, and $99 \%$ semiconductor-enriched SWCNT networks to $\mathrm{NH}_{3}$ gas.

Figure 2 shows the typical current $(I)$-voltage $(V)$ characteristics of three SWCNT-network sensors $(S / M=$ $66 \%, 90 \%$, and $99 \%$ ) recorded at room temperature before exposure to $\mathrm{NH}_{3}$ gas. As expected, all SWCNT-network devices exhibited very linear and symmetric $I-V$ behavior regardless of the value of $S / M$, indicating the good ohmic Pd-SWCNT contacts had been achieved. Javey et al. [32] demonstrated that it is possible to obtain CNTFETs with zero Schottky barrier (SB) height for holes by using Pd contacts on semiconducting CNTs to realize the greatest benefit of SWCNTs. SWCNT devices with reliable ohmic contacts exhibit $I-V$ characteristics that are more similar to highly resistive devices because the transport is not limited by the contacts. The measured resistances for the three devices are $1 \mathrm{k} \Omega, 0.5 \mathrm{k} \Omega$, and $2.4 \mathrm{k} \Omega$, which include the wiring and contact resistances, but the SWCNT-network resistance is the dominant part of the observed sample resistance because of the good ohmic contact. Despite the low $S / M$ ratio of $66 \%$ SWCNT, the initial resistance of the device was higher than that of the $90 \%$ device. As noted in several papers [33, 34], contact resistance is inversely proportional to the length of the CNTs. The reversal of the initial resistance of the $90 \%$ SWCNT device is due to differences in the length of $66 \%$ $(\sim 20 \mathrm{~nm})$ and $90 \% \mathrm{CNT}$ (approximately $400 \mathrm{~nm}$ ) provided by each company.

The $I-V$ curves recorded after exposing three devices to different $\mathrm{NH}_{3}$ concentrations are also highly linear and symmetrical for the $\mathrm{NH}_{3}$ concentrations (3 to $42 \mathrm{ppm}$ ), as illustrated in the insets in Figures 3(a)-3(c). The resistances of these devices were calculated at a bias of $1 \mathrm{~V}$ and plotted as a function of the concentrations in Figures 3(a)-3(c). The unenriched $66 \%$ and $90 \%$ enriched SWCNT sensors exhibited a typical nonlinear characteristic for CNT-based gas sensors $[8,10,35,36]$, whereas the $99 \%$ enriched device exhibited a linear increase in resistance with increasing $\mathrm{NH}_{3}$ gas concentration, as shown in Figure 3(c).

3.2. Comparison of the Sensitivity. Figure 4 shows the calibrated responsiveness $\left[\Delta R / R_{0}=\left(R-R_{0}\right) / R_{0}\right.$, where $R$ is the steady-state resistance of the sensor when exposed to 


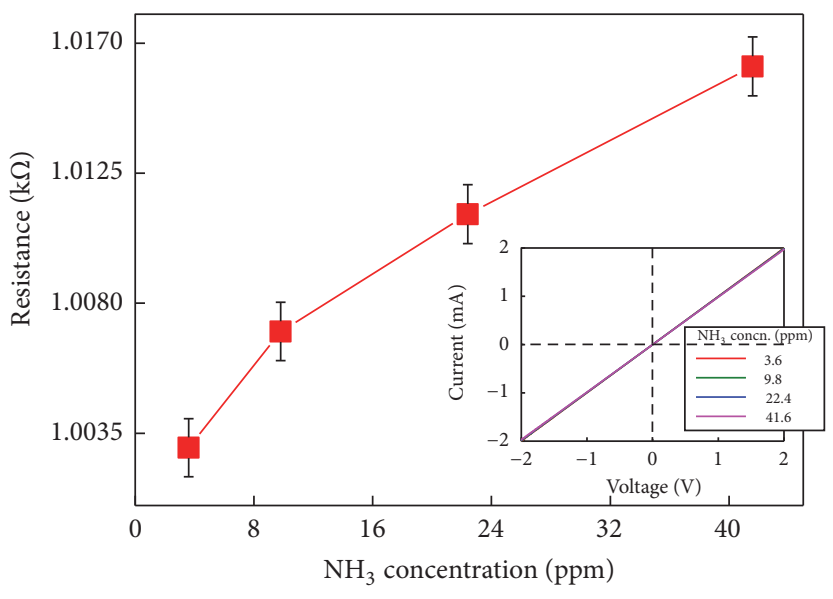

(a) Unenriched $66 \%$ SWCNT

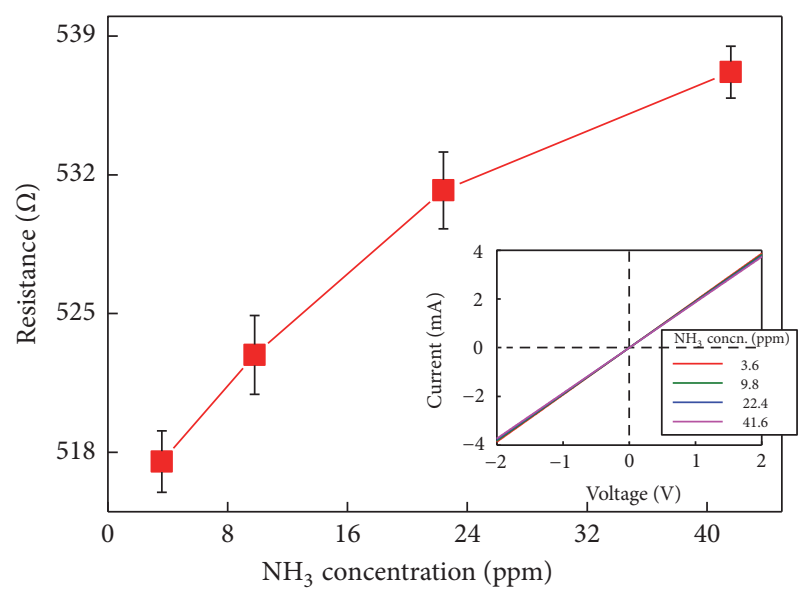

(b) $90 \%$ enriched SWCNT

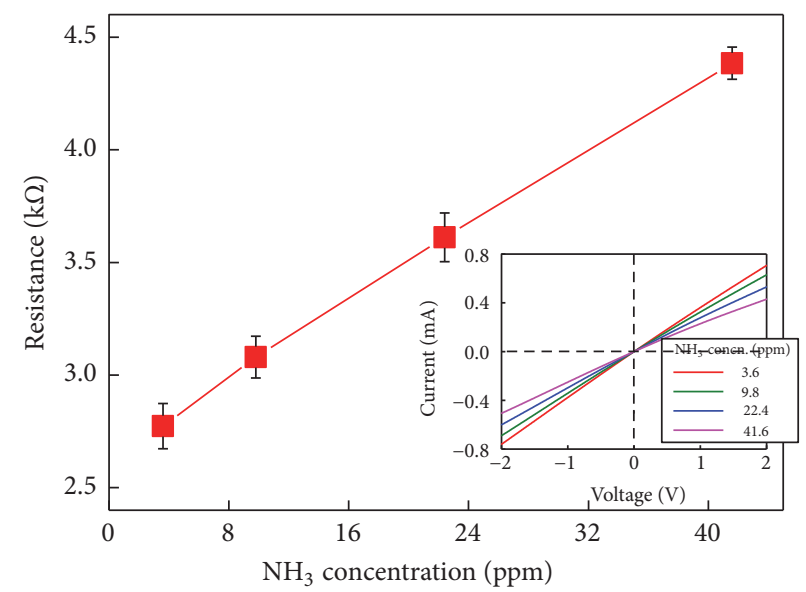

(c) $99 \%$ enriched SWCNT

FIGURE 3: Device resistance as a function of $\mathrm{NH}_{3}$ concentration for the unenriched $66 \%, 90 \%$ enriched, and $99 \%$ enriched SWCNT-network sensors. The insets represent the $I-V$ characteristic curves to different $\mathrm{NH}_{3}$ concentration.

various gas concentrations, and $R_{0}$ is the device resistance in the outgassed state] as a function of the $\mathrm{NH}_{3}$ concentration at room temperature for all investigated sensors in the ammonia-concentration range of 3-42 ppm. A general linear response is observed with increasing $\mathrm{NH}_{3}$ concentration with a significantly greater responsiveness for the $99 \%$ enriched network compared to unenriched $66 \%$ and $90 \%$ enriched films. This suggests that a large $S / M$ is the most important factor for achieving a high sensitivity in mixed SWCNTnetwork-based gas sensors (see the following section).

Figure 5 compares the sensitivity (in \%/ppm $\mathrm{NH}_{3}$ ) calculated from calibration slopes in Figure 4. The unenriched $66 \%$ and $90 \%$ enriched samples exhibited a sensitivity of $0.033 \% / \mathrm{ppm}$ and $0.099 \% / \mathrm{ppm}$, respectively. The most important result is that the $99 \%$ enriched device exhibited a sensitivity 17.7 times higher than the $90 \%$ enriched device.

The semiconducting or metallic properties of SWCNTs stem from the variation of their electronic structure, which is composed of arm-chair and zigzag structure. In previously reported papers, the authors claimed that the charge transfer of $\mathrm{NH}_{3}$ gas does not depend on the structure of the CNTs [37] as well as the fact that the interaction between $\mathrm{NH}_{3}$ gas molecules and nanotube does not have a significant effect on the electronic structures of SWCNTs [38]. Therefore, the unexpected large difference in sensitivity between $90 \%$ and $99 \%$ enriched samples is a consequence of the many $\mathrm{s}$-SWCNTs being shorted by m-SWCNTs. The electrical transport through the SWCNT networks with a low $S / M$ is usually dominated by the metallic pathways because of their low resistivity, as shown in the equivalent electric circuit in the inset of Figure 5. As a result, the s-SWCNTs are shorted by m-SWCNTs; therefore, a few $\mathrm{m}$-SWCNTs in a network can drastically weaken the sensing effect, even for a high enrichment of $90 \% \mathrm{~s}-\mathrm{SWNT}$. This suggests that it is extremely important to have a percolative network consisting of only s-SWCNTs without metallic shorts to achieve high sensitivity.

3.3. Comparison of the Dynamic Response. An overall comparison of the measured response and recovery characteristics of the SWCNT-network sensors is presented in Figures 6(a)-6(c) when they were cycled between $\mathrm{NH}_{3}$ and $\mathrm{N}_{2}$ 


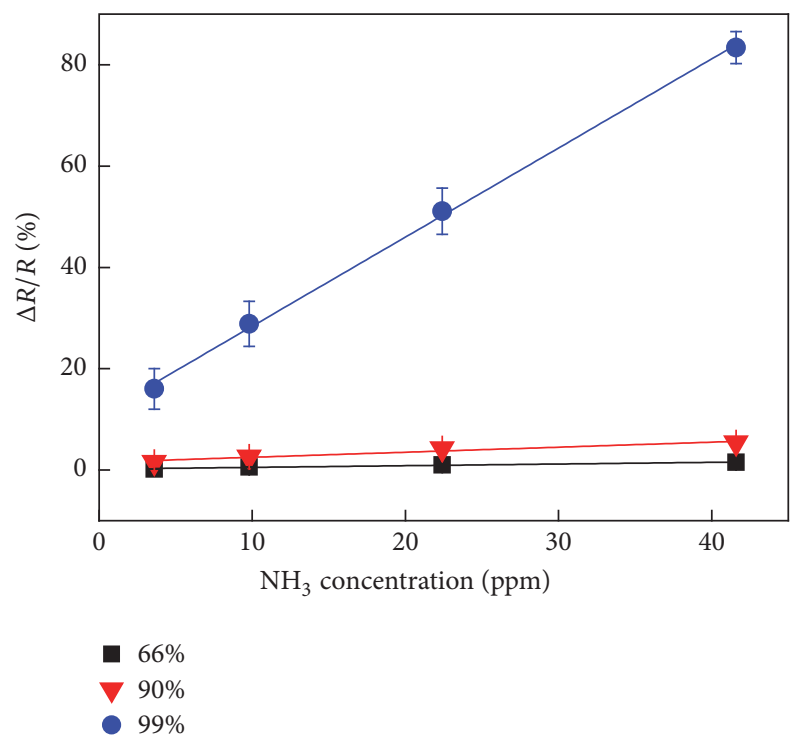

FIGURE 4: Calibration plots of the resistance variation versus the $\mathrm{NH}_{3}$ concentration for the same sensors in Figure 3. The data represent the average of the measurements conducted for at least three independent sensors. The error bars are not indicated for clarity.

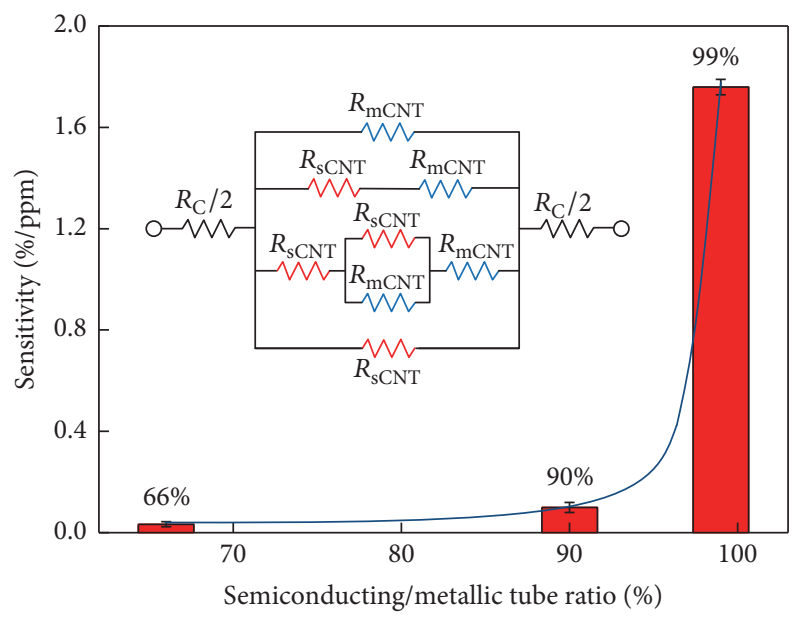

FIGURE 5: Comparison of the sensitivity for the unenriched $66 \%$ and $90 \%$ and $99 \%$ enriched SWCNT-network sensors. The error bars represent the standard deviation of the measurements performed for at least three independent sensors.

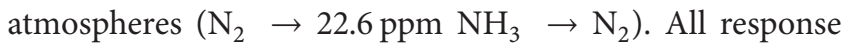
curves are normalized by the resistance $R_{0}$ before exposure to the analyte. As the observations suggest, the sensors demonstrated a long absorption time and furthermore an incomplete recovery of initial resistance during desorption process. They recovered $54 \%$ to $98 \%$ of the response after the $\mathrm{NH}_{3}$ flow was halted.

Table 1 summarizes the measured adsorption ( $90 \%$ point of the final steady-state resistance) and desorption (10\% point of the final steady-state resistance) times for the three devices. The response time was found to be almost the same for the
TABLE 1: Summary of the adsorption and desorption times. Test chamber atmosphere: $\mathrm{N}_{2} \rightarrow 22.6 \mathrm{ppm} \mathrm{NH}_{3} \rightarrow \mathrm{N}_{2}$.

\begin{tabular}{lccc}
\hline Sample & $66 \%$ & $90 \%$ & $99 \%$ \\
\hline Adsorption time (s) & 440 & 442 & 455 \\
Desorption time (s) & $\mathrm{x}$ & $\mathrm{x}$ & 1039 \\
\hline
\end{tabular}

three sensors. However, the response time for each device degraded with increasing $\mathrm{NH}_{3}$ concentration. This perhaps indicates slow charge transfer from the $\mathrm{NH}_{3}$ molecules to the SWCNTs due to the gradual transition from monolayer to multilayer adsorption as the concentration increases. In addition, the devices with low $S / M$ recovered more slowly compared to high $S / M$ sensors. This may be attributed to the difference in the ammonia molecule-CNT binding mechanisms or energy. Several works [39-41] have showed that $\mathrm{NH}_{3}$ molecules may be absorbed into SWCNTs via both physisorption and chemisorption. An s-SWCNT has more sites for chemisorption than an m-SWCNT. When switching from an $\mathrm{NH}_{3}$ atmosphere to a $\mathrm{N}_{2}$ atmosphere, the $\mathrm{NH}_{3}$ molecules very weakly physisorbed on the surface are easily released by the kinetic energy of the incoming $\mathrm{N}_{2}$ gas molecules, leading to a reversible change in the device resistance. The chemisorbed molecules, however, cannot be broken by the $\mathrm{N}_{2}$ gas flow alone, causing a larger buildup in chemisorbed $\mathrm{NH}_{3}$ on the m-SWCNT surface and thus an irreversible change in the resistance [42].

3.4. Effect of the Oxygen Plasma Treatment on the Networks. The surface chemical composition of the SWCNTs has a strong influence on the gas absorption process and therefore the sensing properties. In previous works [24, 43], an $\mathrm{O}_{2}$ plasma treatment was found to introduce a number of oxygen-containing defects into the surface of the CNT film, and these functional groups are known to significantly increase the sensitivity to gases. Although many workers have studied CNT gas sensors functionalized using a plasma process, no reported effects of a plasma treatment on the SWCNT networks with different $S / M$ were found in the literature. The unenriched $66 \%$ and $90 \%$ enriched SWCNTnetwork sensors were only tested because the 99\% enriched SWCNT-network sensor was completely etched away, even under very low power $\mathrm{O}_{2}$ plasma.

Figure $7(\mathrm{a})$ is full-scale XPS spectra for $66 \%$ and $90 \%$ s-SWCNT before and after plasma treatment. Based on previously reported work [24], the Cls peak is generally shown at about $285 \mathrm{eV}$, and the typical O1s peak appears at approximately $533.4 \mathrm{eV}$. Before the plasma treatment, the $\mathrm{O} / \mathrm{C}$ ratio for the $90 \% \mathrm{~s}$-SWCNT sample $(178.92 \%)$ is very larger compared to that of the $66 \%$ s-SWCNT sample $(36.86 \%)$. $\mathrm{High} \mathrm{O} / \mathrm{C}$ ratio contributes to the resistance change of the SWCNT film by the charge transfer induced by adsorption of $\mathrm{NH}_{3}$ molecules because polar $\mathrm{NH}_{3}$ molecules form strong hydrogen bonds with the oxygen atoms on the oxidized tube. After plasma treatment, the $\mathrm{O} / \mathrm{C}$ ratio increased for both the $66 \%$ and $90 \%$ s-SWCNT samples, and especially the variation for $66 \%$ sample was dramatically large. These changes 


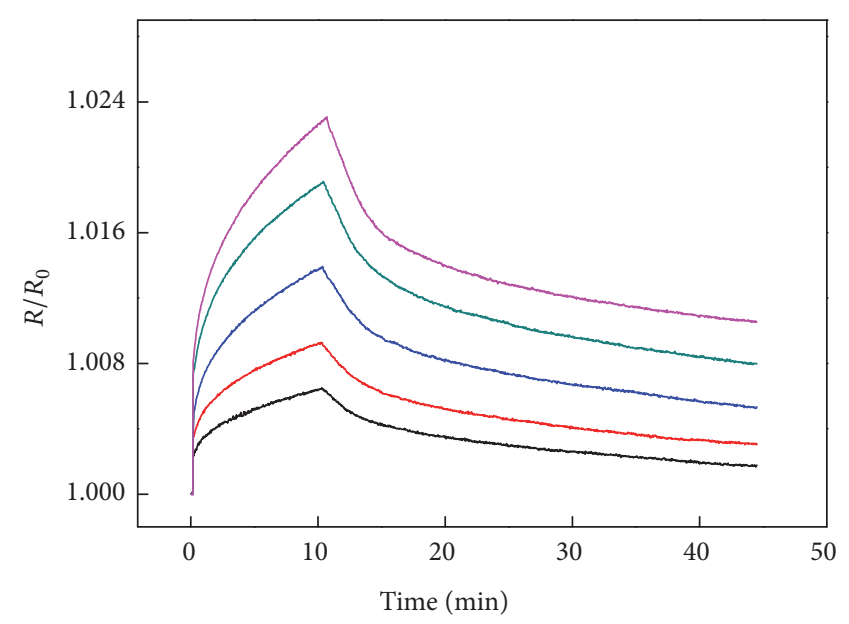

$\mathrm{NH}_{3}$ concn. (ppm)
$-3.6 \quad 41.6$
$-9.8 \quad-64.2$
-22.4

(a)

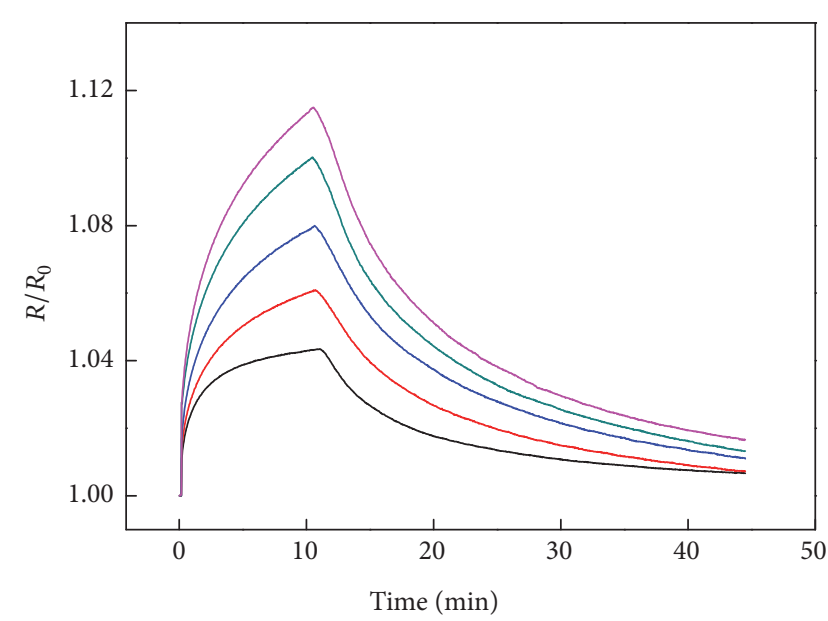

$\mathrm{NH}_{3}$ concn. (ppm)

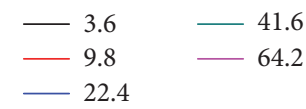

(b)

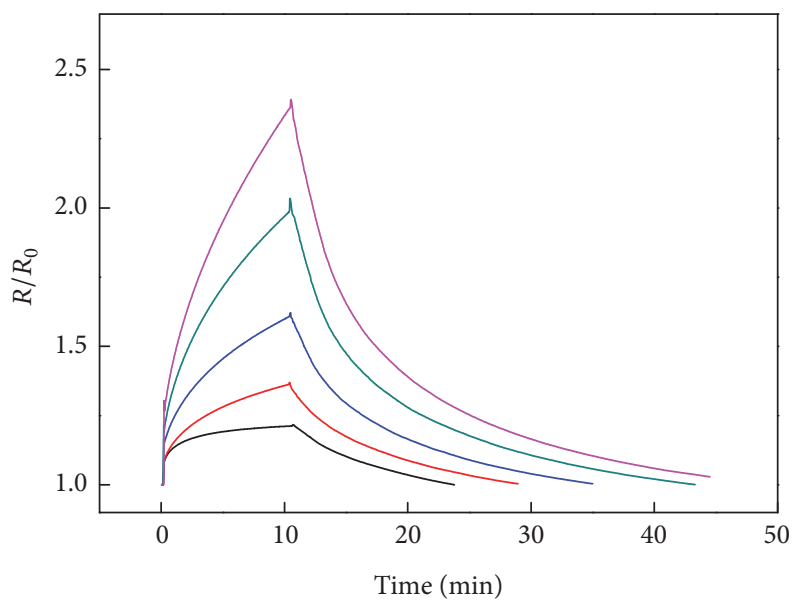

$\mathrm{NH}_{3}$ concn. (ppm)
$-3.6 \quad 41.6$
$-9.8 \quad-64.2$
-22.4

(c)

FIGURE 6: Responses of the (a) unenriched $60 \%$ and (b) $90 \%$ and (c) $99 \%$ enriched SWCNT-network sensors to different $\mathrm{NH}_{3}$ concentrations at room temperature. $R_{0}$ and $R$ are the resistances before and after exposure to the analyte.

contribute significantly to the sensitivity and response time of the device.

Figure 8 shows the measured dynamic responses of the unenriched $66 \%$ and $90 \%$ enriched SWCNT-network sensors, and the reproducible responses measured at the same $\mathrm{NH}_{3}$ gas concentration are presented in Figure S2. After plasma treatment, both p-66\% and p-90\% samples exhibited a significant improvement in the responsiveness and response time compared to the equivalent untreated sensors.

Figures 9(a) and 9(b) compare the normalized sensitivity (in \%/ppm $\mathrm{NH}_{3}$ ) and response time before and after plasma treatment for the unenriched $66 \%$ and $90 \%$ enriched devices.
It is observed from Figure 9(a) that the amount of s-SWCNT content and the plasma activation significantly affect the sensitivity of the SWCNT gas sensors. The p-66\% samples exhibited an increase in sensitivity from 0.027 to $0.142 \% / \mathrm{ppm}$ after the plasma treatment. Furthermore, the p-90\% enriched devices exhibited a sensitivity of $1.521 \% / \mathrm{ppm}$, which is approximately equal to an improvement in sensitivity of 13.4 times the untreated $90 \%$ enriched samples $(0.113 \% / \mathrm{ppm})$ and 10.7 times the p-66\% samples (see Figure SI, in Section S3). In addition, the two plasma-treated devices exhibited a large reduction in the response time of $70-78 \%$, as shown in Figure 9(b). The significantly enhanced performance with 


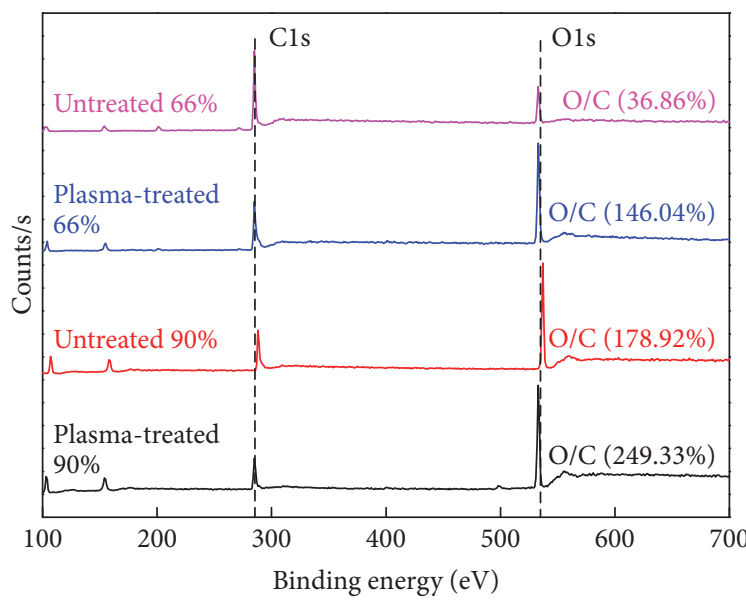

(a)

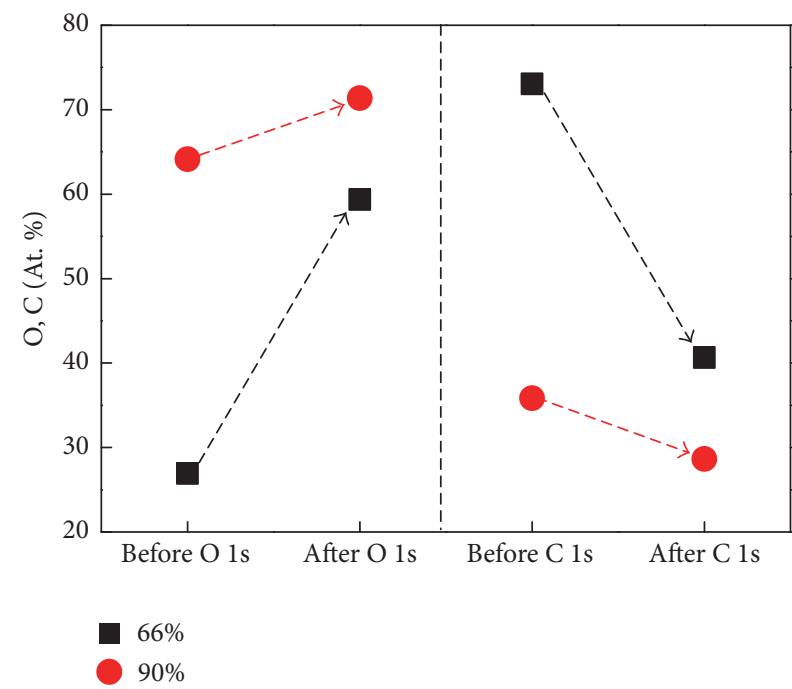

(b)

FIGURE 7: (a) Full-scale XPS spectra of $66 \%$ and $90 \%$ enriched SWCNT films and (b) the calculated atomic percentage of O1s and C1s before and after the $\mathrm{O}_{2}$ plasma treatment. The optimum conditions of plasma treatment for each CNT were determined experimentally.

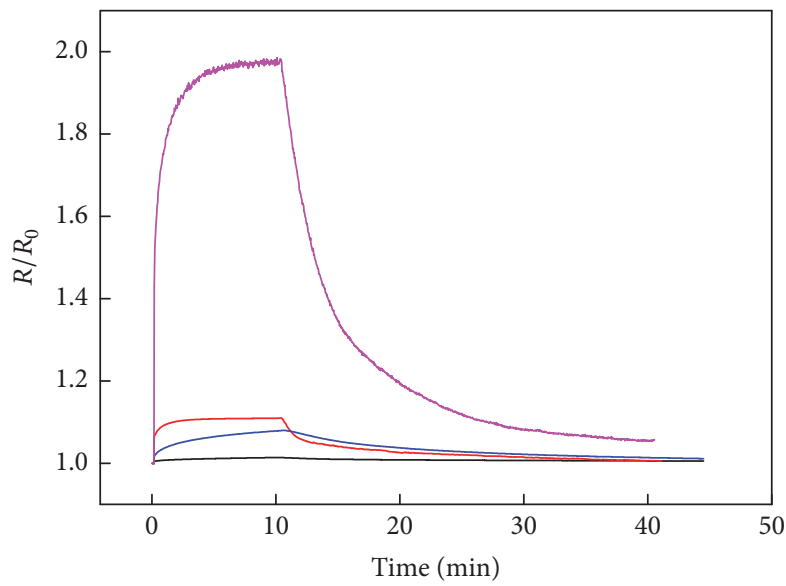

$$
\begin{array}{ll}
90 \% \text { SWCNT } & 66 \% \text { SWCNT } \\
- \text { Before treatment } & - \text { Before treatment } \\
\text { After treatment } & - \text { After treatment }
\end{array}
$$

FIGURE 8: Responses before and after plasma treatment of the unenriched $60 \%$ and $90 \%$ enriched SWCNT-network sensors to an $\mathrm{NH}_{3}$ concentration of $22.6 \mathrm{ppm}$ at room temperature. $R_{0}$ and $R$ are the resistances before and after exposure to the analyte.

the oxygen plasma-treated devices is related to the nature of the SWCNT surface. The plasma-treated SWCNTs have more oxygen-containing functional groups, especially carboxyl groups, which act as sites for gas-molecule chemisorption $[16,19]$. Such anchoring sites formed on the CNT surface are known to significantly increase the electronic interaction of CNTs with gas molecules. This affects the SWCNT-network in two ways: faster charge transfer from the $\mathrm{NH}_{3}$ molecules adsorbed on the defect sites to the SWCNT bulk, causing a faster response time, or hole-carrier depletion [20], causing an increase in resistance $(\Delta R)$ and thus sensitivity. The enhancement of sensitivity is much more pronounced for the p-90\% enriched devices, suggesting that the semiconducting SWCNT-network is very sensitive to oxygen-containing functional groups, as compared to the metallic SWCNTs. In addition, the plasma-treated devices with high $S / M$ showed more slow response time than that with low $S / M$. Although some researchers argue that $\mathrm{m}$-SWCNTs with chemisorption sites increase response time [44], our result for concurrent reaction of s- and m-SWCNT may be attributed to the reason why the electrical properties of $\mathrm{m}$-SWCNTs are relatively insensitive to the interaction with $\mathrm{NH}_{3}$ [15].

\section{Conclusions}

In this work, we explored the influence of varying $S / M$ and an oxygen plasma treatment on the static and dynamic characteristics of $\mathrm{NH}_{3}$ gas sensors based on randomly oriented mixed SWCNT networks. We found that there is a close relationship between $S / M$ of the SWCNT networks and the sensitivity of gas sensors based on these networks. The most important result is that the $99 \%$ semiconductor-enriched devices exhibit a much higher sensitivity compared to the 90\% enriched device, suggesting that a few m-SWCNTs in a network can drastically weaken the sensing effect, even for high enrichment, and a percolative network consisting of only s-SWCNTs without metallic shorts is required to achieve high performance. We also demonstrated the effect of an oxygen plasma treatment on the highly semiconductorenriched SWCNT-network sensors. After an oxygen plasma treatment, the 90\% enriched SWCNT-network exhibited a much higher sensitivity to $\mathrm{NH}_{3}$ gas than unenriched $66 \%$ and pristine $90 \%$ enriched samples, showing that the $\mathrm{O}_{2}$ plasma treatment has a stronger influence on the semiconductorenriched SWCNT-network than the normal unenriched network, possibly indicating that more oxygenated defects are 


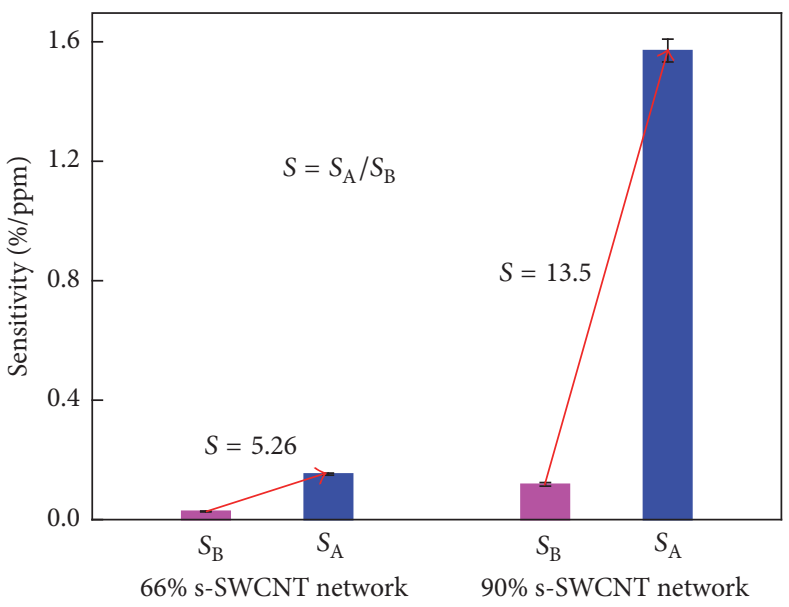

$S_{\mathrm{B}}$ : sensitivity before treatment $S_{\mathrm{A}}$ : sensitivity after treatment

(a)

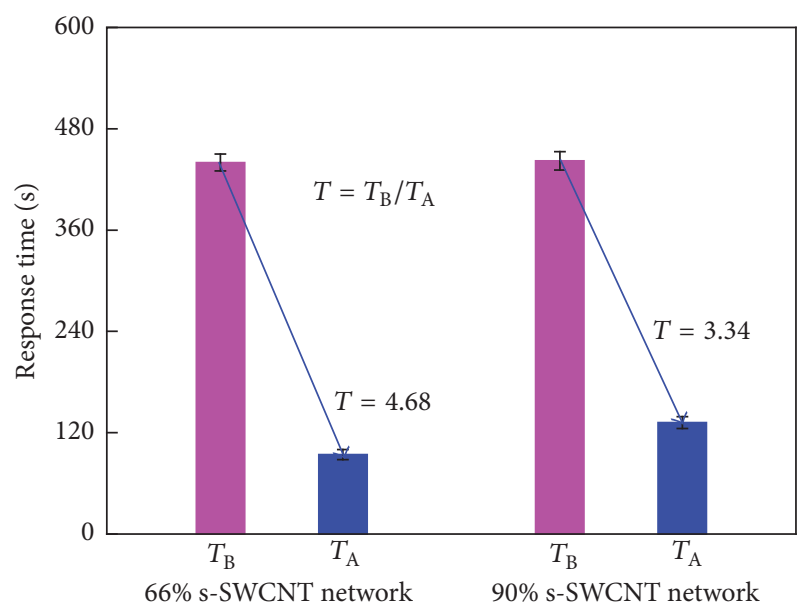

$T_{\mathrm{B}}$ : response time before treatment $T_{\mathrm{A}}$ : response time after treatment

(b)

Figure 9: Comparison of the (a) sensitivity and (b) response time before and after plasma treatment for the unenriched $66 \%$ and $90 \%$ enriched SWCNT-network sensors. The response time is the $90 \%$ point of the final steady-state resistance. The error bars represent the standard deviation of measurements performed for at least three independent sensors.

created in the s-SWCNT-network than in the m-SWCNTnetwork. These results provide valuable insights into the role of $S / M$ and the plasma treatment towards achieving a large improvement in the performance of SWCNT-network gas sensors.

\section{Conflicts of Interest}

The authors declare that there are no conflicts of interest regarding the publication of this paper.

\section{Acknowledgments}

This study was supported by 2016 Research Grant from Kangwon National University (no. 520160103) and by Basic Science Research Program through the National Research Foundation of Korea (NRF) funded by the Ministry of Education (NRF-2015R1A6A3A04060326).

\section{References}

[1] P. K. Sekhar, E. L. Brosha, R. Mukundan, and F. H. Garzon, "Chemical sensors for environmental monitoring and homeland security," The Electrochemical Society Interface, vol. 19, no. 4, pp. 35-40, 2010.

[2] R. D. van Zee and G. S. Pomrenke, "Nanotechnology enabled sensing: report of the national nanotechnology initiative workshop," 2009, http://www.nano.gov/sites/default/files/NNI-Nanosensors-stdres.pdf.

[3] S. K. Vashist, D. Zheng, K. Al-Rubeaan, J. H. T. Luong, and F.S. Sheu, "Advances in carbon nanotube based electrochemical sensors for bioanalytical applications," Biotechnology Advances, vol. 29, no. 2, pp. 169-188, 2011.
[4] T. Zhang, S. Mubeen, N. V. Myung, and M. A. Deshusses, "Recent progress in carbon nanotube-based gas sensors," Nanotechnology, vol. 19, no. 33, Article ID 332001, 2008.

[5] R. A. Potyrailo, C. Surman, N. Nagraj, and A. Burns, "Materials and transducers toward selective wireless gas sensing," Chemical Reviews, vol. 111, no. 11, pp. 7315-7354, 2011.

[6] M. E. Roberts, M. C. Lemieux, and Z. Bao, "Sorted and aligned single-walled carbon nanotube networks for transistor-based aqueous chemical sensors," ACS Nano, vol. 3, no. 10, pp. 3287$3293,2009$.

[7] K. A. Mirica, J. M. Azzarelli, J. G. Weis, J. M. Schnorr, and T. M. Swager, "Rapid prototyping of carbon-based chemiresistive gas sensors on paper," Proceedings of the National Academy of Sciences of the United States of America, vol. 110, no. 35, pp. E3265-E3270, 2013.

[8] J. Li, Y. Lu, Q. Ye, M. Cinke, J. Han, and M. Meyyappan, “Carbon nanotube sensors for gas and organic vapor detection," Nano Letters, vol. 3, no. 7, pp. 929-933, 2003.

[9] J. Zhang, A. Boyd, A. Tselev, M. Paranjape, and P. Barbara, "Mechanism of $\mathrm{NO}_{2}$ detection in carbon nanotube field effect transistor chemical sensors," Applied Physics Letters, vol. 88, no. 12, Article ID 123112, 2006.

[10] N. Peng, Q. Zhang, C. L. Chow, O. K. Tan, and N. Marzari, "Sensing mechanisms for carbon nanotube based $\mathrm{NH}_{3}$ gas detection," Nano Letters, vol. 9, no. 4, pp. 1626-1630, 2009.

[11] J. Kong, M. G. Chapline, and H. Dai, "Functionalized Carbon Nanotubes for Molecular Hydrogen Sensors," Advanced Materials, vol. 13, no. 18, pp. 1384-1386, 2001.

[12] K. Bradley, J.-C. P. Gabriel, A. Star, and G. Grüner, "Shortchannel effects in contact-passivated nanotube chemical sensors," Applied Physics Letters, vol. 83, no. 18, pp. 3821-3823, 2003.

[13] E. S. Snow and F. K. Perkins, "Capacitance and conductance of single-walled carbon nanotubes in the presence of chemical vapors," Nano Letters, vol. 5, no. 12, pp. 2414-2417, 2005. 
[14] E. S. Snow, F. K. Perkins, E. J. Houser, S. C. Badescu, and T. L. Reinecke, "Chemical detection with a single-walled carbon nanotube capacitor," Science, vol. 307, no. 5717, pp. 1942-1945, 2005.

[15] J. Kong, N. R. Franklin, C. Zhou et al., "Nanotube molecular wires as chemical sensors," Science, vol. 287, no. 5453, pp. 622$625,2000$.

[16] J. Kong and H. Dai, "Full and modulated chemical gating of individual carbon nanotubes by organic amine compounds," Journal of Physical Chemistry B, vol. 105, no. 15, pp. 2890-2893, 2001.

[17] M. Grujicic, G. Cao, and R. Singh, "The effect of topological defects and oxygen adsorption on the electronic transport properties of single-walled carbon-nanotubes," Applied Surface Science, vol. 211, no. 1-4, pp. 166-183, 2003.

[18] L. Valentini, F. Mercuri, I. Armentano et al., "Role of defects on the gas sensing properties of carbon nanotubes thin films: experiment and theory," Chemical Physics Letters, vol. 387, no. 4-6, pp. 356-361, 2004.

[19] J. A. Robinson, E. S. Snow, Ş. C. Badescu, T. L. Reinecke, and F. K. Perkins, "Role of defects in single-walled carbon nanotube chemical sensors," Nano Letters, vol. 6, no. 8, pp. 1747-1751, 2006.

[20] P. C. P. Watts, N. Mureau, Z. Tang, Y. Miyajima, J. David Carey, and S. R. P. Silva, "The importance of oxygen-containing defects on carbon nanotubes for the detection of polar and non-polar vapours through hydrogen bond formation," Nanotechnology, vol. 18, no. 17, Article ID 175701, 2007.

[21] J. Zhang, H. Zou, Q. Qing et al., "Effect of chemical oxidation on the structure of single-walled carbon nanotubes," Journal of Physical Chemistry B, vol. 107, no. 16, pp. 3712-3718, 2003.

[22] A. Felten, J. Ghijsen, J.-J. Pireaux et al., "Effect of oxygen rfplasma on electronic properties of CNTs," Journal of Physics D: Applied Physics, vol. 40, no. 23, pp. 7379-7382, 2007.

[23] A. Felten, C. Bittencourt, J. J. Pireaux, G. Van Lier, and J. C. Charlier, "Radio-frequency plasma functionalization of carbon nanotubes surface $\mathrm{O}_{2}, \mathrm{NH}_{3}$, and $\mathrm{CF}_{4}$ treatments," Journal of Applied Physics, vol. 98, no. 7, Article ID 074308, 2005.

[24] J. H. Kim, M.-J. Song, C. J. Lee, J.-H. Lee, J.-H. Kim, and N. K. Min, "A comparative study of electrochemical and biointerfacial properties of acid- and plasma-treated single-walled carbonnanotube-film electrode systems for use in biosensors," Carbon, vol. 52, pp. 398-407, 2013.

[25] C. Wang, K. Takei, T. Takahashi, and A. Javey, "Carbon nanotube electronics-moving forward," Chemical Society Reviews, vol. 42, no. 7, pp. 2592-2609, 2013.

[26] C. Wang, J. Zhang, and C. Zhou, "Macroelectronic integrated circuits using high-performance separated carbon nanotube thin-film transistors," ACS Nano, vol. 4, no. 12, pp. 7123-7132, 2010.

[27] M. Engel, J. P. Small, M. Steiner et al., “Thin film nanotube transistors based on self-assembled, aligned, semiconducting carbon nanotube arrays," ACS Nano, vol. 2, no. 12, pp. 24452452, 2008

[28] N. Rouhi, D. Jain, and P. J. Burke, "High-performance semiconducting nanotube inks: progress and prospects," ACS Nano, vol. 5, no. 11, pp. 8471-8487, 2011.

[29] C. Wang, J.-C. Chien, K. Takei et al., "Extremely bendable, highperformance integrated circuits using semiconducting carbon nanotube networks for digital, analog, and radio-frequency applications," Nano Letters, vol. 12, no. 3, pp. 1527-1533, 2012.
[30] N. Rouhi, D. Jain, K. Zand, and P. J. Burke, "Fundamental limits on the mobility of nanotube-based semiconducting inks," Advanced Materials, vol. 23, no. 1, pp. 94-99, 2011.

[31] K.-P. Yoo, K.-H. Kwon, N.-K. Min, M. J. Lee, and C. J. Lee, "Effects of $\mathrm{O}_{2}$ plasma treatment on $\mathrm{NH}_{3}$ sensing characteristics of multiwall carbon nanotube/polyaniline composite films," Sensors and Actuators, B: Chemical, vol. 143, no. 1, pp. 333-340, 2009.

[32] A. Javey, J. Guo, Q. Wang, M. Lundstrom, and H. Dai, "Ballistic carbon nanotube field-effect transistors," Nature, vol. 424, no. 6949, pp. 654-657, 2003.

[33] C. Lan, P. Srisungsitthisunti, P. B. Amama, T. S. Fisher, X. $\mathrm{Xu}$, and $\mathrm{R}$. G. Reifenberger, "Measurement of metal/carbon nanotube contact resistance by adjusting contact length using laser ablation," Nanotechnology, vol. 19, no. 12, 2008.

[34] S. Li, Z. Yu, C. Rutherglen, and P. J. Burke, "Electrical properties of $0.4 \mathrm{~cm}$ long single-walled carbon nanotubes," Nano Letters, vol. 4, no. 10, pp. 2003-2007, 2004.

[35] C. Wei, L. Dai, A. Roy, and T. B. Tolle, "Multifunctional chemical vapor sensors of aligned carbon nanotube and polymer composites," Journal of the American Chemical Society, vol. 128, no. 5, pp. 1412-1413, 2006.

[36] F. Rigoni, S. Tognolini, P. Borghetti et al., "Enhancing the sensitivity of chemiresistor gas sensors based on pristine carbon nanotubes to detect low-ppb ammonia concentrations in the environment," Analyst, vol. 138, no. 24, pp. 7392-7399, 2013.

[37] J. Zhao, H. Park, J. Han, and J. P. Lu, "Electronic properties of carbon nanotubes with covalent sidewall functionalization," Journal of Physical Chemistry B, vol. 108, no. 14, pp. 4227-4230, 2004.

[38] J. Zhao, A. Buldum, J. Han, and J. P. Lu, "Gas molecule adsorption in carbon nanotubes and nanotube bundles," Nanotechnology, vol. 13, no. 2, pp. 195-200, 2002.

[39] K. Bradley, J.-C. P. Gabriel, M. Briman, A. Star, and G. Grüner, "Charge transfer from ammonia physisorbed on nanotubes," Physical Review Letters, vol. 91, no. 21, p. 218301, 2003.

[40] M. D. Ellison, M. J. Crotty, D. Koh, R. L. Spray, and K. E. Tate, "Adsorption of $\mathrm{NH}_{3}$ and $\mathrm{NO}_{2}$ on single-walled carbon nanotubes," Journal of Physical Chemistry B, vol. 108, no. 23, pp. 7938-7943, 2004.

[41] J. Andzelm, N. Govind, and A. Maiti, "Nanotube-based gas sensors-role of structural defects," Chemical Physics Letters, vol. 421, no. 1-3, pp. 58-62, 2006.

[42] K. G. Ong, K. Zeng, and C. A. Grimes, "A wireless, passive carbon nanotube-based gas sensor," IEEE Sensors Journal, vol. 2, no. 2, pp. 82-88, 2002.

[43] J. H. Kim, J.-H. Jin, J.-Y. Lee, E. J. Park, and N. K. Min, “Covalent attachment of biomacromolecules to plasma-patterned and functionalized carbon nanotube-based devices for electrochemical biosensing," Bioconjugate Chemistry, vol. 23, no. 10, pp. 2078-2086, 2012.

[44] A. Ricca and C. W. Bauschlicher Jr., "The adsorption of $\mathrm{NO}_{2}$ on $(9,0)$ and $(10,0)$ carbon nanotubes," Chemical Physics, vol. 323, no. 2-3, pp. 511-518, 2006. 

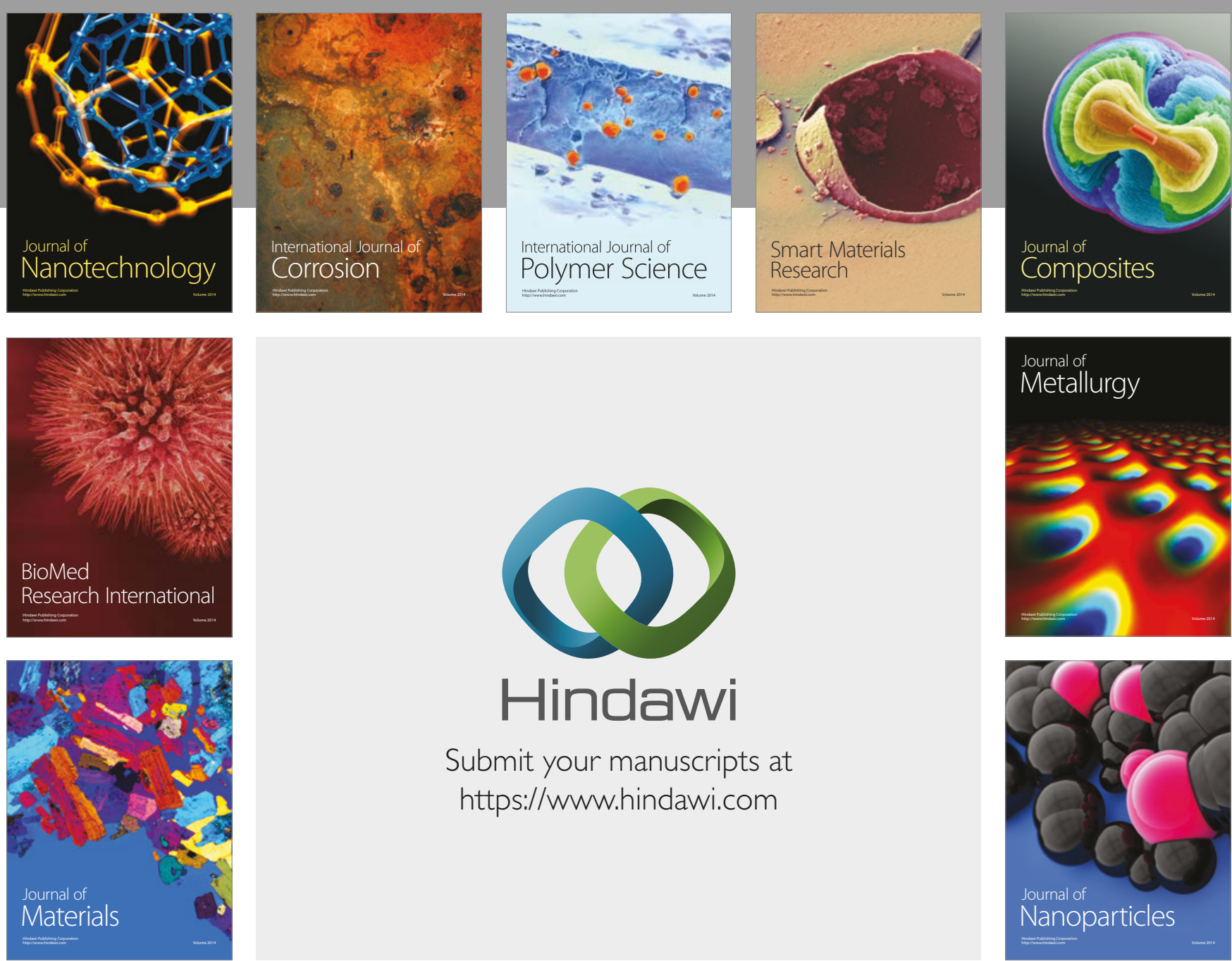

\section{Hindawi}

Submit your manuscripts at

https://www.hindawi.com
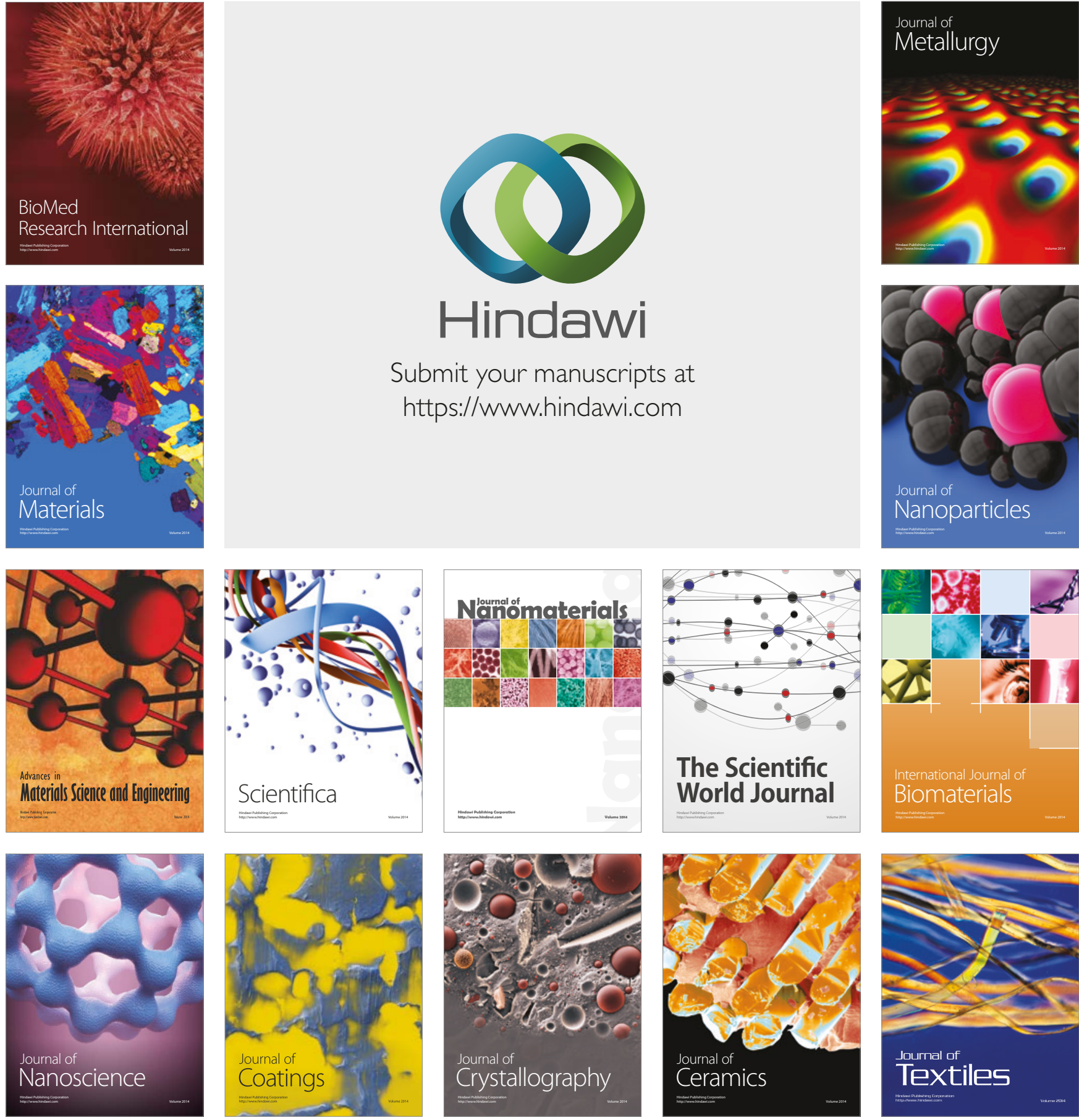

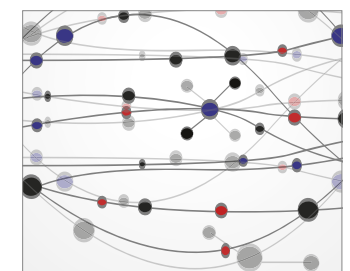

The Scientific World Journal
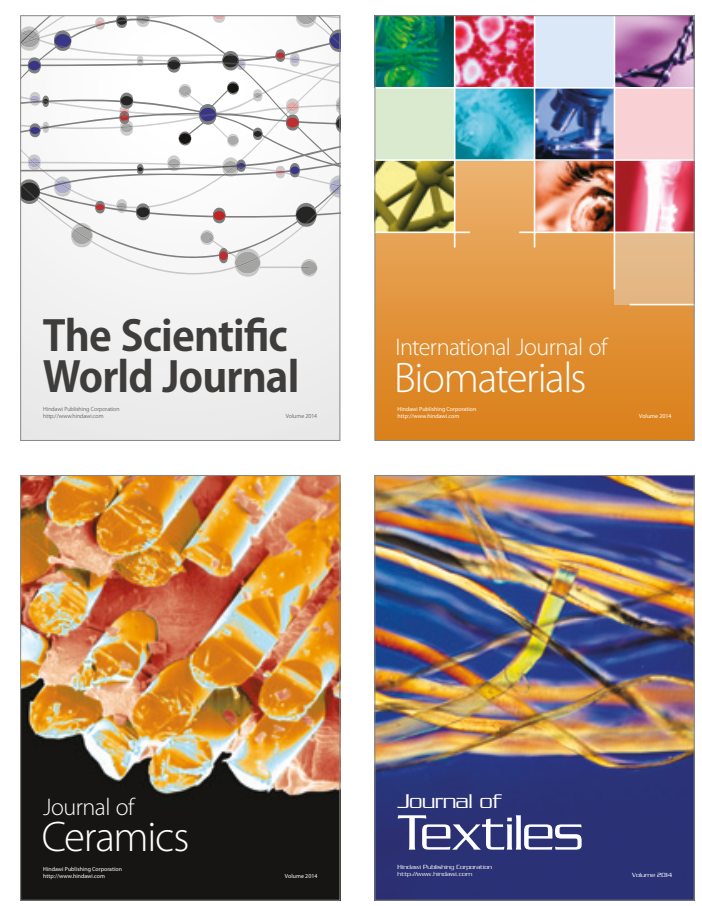